\title{
Determinantes do grau de aceitabilidade da dieta hospitalar: ferramentas para a prática clínica?
}

Determinants of acceptability degree of the hospital diet: tools for clinical practice?

Simone Augusta Ribas'

Elaine de Oliveira Pinto ${ }^{1}$

Camila Batista Rodrigues ${ }^{2}$

I Nutricionista, Divisão de Nutrição, Hospital Universitário Pedro Ernesto. Rio de Janeiro, RJ, Brasil.

1 Nutricionista, Residente de Nutrição, Divisão de Nutrição, Hospital Universitário Pedro Ernesto. Rio de Janeiro, RJ, Brasil.

${ }^{2}$ Nutricionista, Universidade Federal do Rio de Janeiro. Rio de Janeiro, RJ, Brasil.

Correspondência / Corrrespondence Simone Augusta Ribas

E-mail: ribasnut@yahoo.com.br

\section{Resumo}

O propósito do estudo foi investigar o grau de aceitabilidade da dieta hospitalar em pacientes internados em um hospital público universitário na cidade do Rio de Janeiro. Trata-se de estudo transversal, no qual aspectos quantitativos e ambientais relacionados a dieta, tempo médio de internação e qualidade de atendimento foram investigados. Os resultados revelaram que a avaliação da aceitação da dieta hospitalar foi considerada de boa a ótima na maioria dos casos e que $88,7 \%$ dos pacientes ingeriram mais da metade do conteúdo total da refeição oferecida. Nas pequenas refeições, as dietas com restrição de nutrientes, incluindo a hipossódica, apresentaram maior grau de aceitação entre seus usuários do que as dietas básicas e com sal. A temperatura inadequada da dieta servida foi o fator que mais contribuiu negativamente para a aceitação alimentar, seguido da falta de intervalo entre o jantar e a ceia. A aceitação da dieta também foi inversamente proporcional ao tempo de internação. Conclui-se que a avaliação da dieta hospitalar deve ser realizada de forma contínua e abrangente, para que os fatores que influenciam na satisfação do usuário possam ser detectados e modificados antes que comprometam seu estado nutricional e fisiopatológico.

Palavras-chave: Dieta. Desnutrição. Pacientes internados. 


\section{Abstract}

This study aimed to investigate the degree of acceptability of the diet among hospital inpatients at a university hospital in the city of Rio de Janeiro. In this cross-sectional study were investigated quantitative and environmental aspects related to diet, mean hospital stay and quality of care. Results showed that assessment of the acceptance of the hospital diet was considered as good to very good in most cases and $88.7 \%$ of patients took more than half of the total content of the meal provided. In small meals, restricted diet nutrients, including low sodium, showed higher degree of acceptance among its users than the basic diets and salt. Inadequate temperature was the most negative factor for food acceptance, followed by short interval between dinner and supper. The acceptance of the diet was also inversely proportional to the length of stay. We conclude that the evaluation of hospital diet should be carried out continuously and comprehensively, so that the factors that influence the user's satisfaction can be detected and modified before they compromise their nutritional status and patho-physiology.

Key words: Diet. Malnutrition. Inpatient.

\section{Introdução}

A prevalência de desnutrição em ambiente hospitalar alcança percentuais que oscilam entre $20 \%$ e $50 \%$, que a caracterizam como importante problema de saúde pública. ${ }^{1,2}$ Sua ocorrência está associada ao aumento da taxa de morbimortalidade, ao período de internação e às despesas hospitalares. ${ }^{3,4}$ Além da doença, alguns trabalhos, ${ }^{5}$ incluindo um estudo multicêntrico, ${ }^{3}$ citam o ambiente hospitalar, as restrições dietéticas e a forma de atendimento como alguns dos fatores que podem comprometer a aceitação da dieta e, por consequência, levar ao quadro de desnutrição intra-hospitalar, ${ }^{6,7}$ reforçando a necessidade da avaliação contínua e criteriosa desses quesitos por parte dos profissionais de saúde.

Apesar da percepção do valor da dieta, que para o paciente está estritamente relacionada à cura de sua doença, o papel da alimentação vai além de suprir calorias e auxiliar na recuperação/ manutenção do seu estado nutricional., ${ }^{8,9}$ Ela também tem caráter afetivo, pois possui o propósito de atenuar o sofrimento gerado no período de internação, em que o indivíduo se desloca de suas atividades e dos papéis desempenhados na família, na comunidade e nas relações de trabalho. ${ }^{10}$ 
Frente à importância dos aspectos sensoriais e simbólicos na função terapêutica da dieta, parte dos hospitais privados ou públicos terceirizados já busca diferenciar seu atendimento e apresentação das preparações culinárias, por meio de uma integração relacionada aos princípios dietéticos e à gastronomia, a fim de desmistificar a ideia da comida sem graça e sem sabor do hospital e melhorar a aceitação da dieta hospitalar. ${ }^{11,12}$

Apesar disso, poucos estudos avaliam a aceitação das dietas orais básicas ou com restrição de algum nutriente em suas preparações culinárias no contexto hospitalar, ${ }^{4}$ e os existentes não a avaliam por tipo de refeição ou não consideram, em suas análises, os fatores sociais e ambientais no processo de aceitação da dieta. Deste modo, o presente trabalho teve como finalidade investigar o grau de aceitabilidade da dieta hospitalar e possíveis fatores de interferência, por meio da percepção dos pacientes, durante o período de internação dentro do hospital universitário.

\section{Métodos}

Participaram do estudo transversal pacientes com idade entre 18 e 70 anos, internados no Hospital Universitário Pedro Ernesto (HUPE) da Universidade do Estado do Rio de Janeiro, localizado na cidade do Rio de Janeiro, no período de agosto a dezembro de 2010.

O HUPE conta com 525 leitos distribuídos em unidades clínicas, especializadas, cirúrgicas, pediátricas e de maternidade, além do centro de terapia intensiva. A unidade de alimentação e nutrição do hospital distribui cerca de 1.980 refeições/dia, sendo $60 \%$ produzidos na instituição e $40 \%$ transportados em recipientes térmicos (dietas normal e branda, com ou sem sal), que atendem a pacientes, acompanhantes e funcionários autorizados. O sistema de porcionamento das refeições é centralizado, mas as dietas são acondicionadas em embalagens de alumínio e os carrinhos de transporte não são térmicos.

Com a finalidade de atender aos propósitos do estudo, foram aceitos os seguintes critérios de inclusão: indivíduos lúcidos e orientados, internados há mais de 48 horas, sem problemas de mastigação, deglutição e alteração de paladar, recebendo dietas de consistência normal a pastosa. Quanto ao aspecto qualitativo, as dietas foram denominadas como básicas (normal, branda e pastosa) e modificadas (hipolipídica, diabético, hiperproteica e hipercalórica, hipoproteica e sem-resíduo). Foram impossibilitados de participar da pesquisa: pacientes em terapia intensiva, com problemas neurológicos e neutropênicos, como também aqueles que apresentaram graves desordens gastrointestinais ou quadro de inapetência, e os acompanhantes.

O universo a ser investigado foi determinado pelo levantamento do número médio de pacientes internados por leito ativo registrado no sistema da Central de Internação do hospital e do número de dietas computadas na estatística da Divisão de Nutrição que atendesse aos critérios desta pesquisa. Desse modo, o cálculo do tamanho da amostra foi efetuado levando em consideração o 
universo de 198 pacientes, um nível de confiança de 95\% e um erro amostral de 5\%, perfazendo um total mínimo de 125 pacientes a serem estudados.

Dados sociais, clínicos e dietéticos foram obtidos do prontuário dos pacientes. Para facilitar a análise, as patologias apresentadas pelos pacientes foram agrupadas em quatro subgrupos: doenças crônicas; doenças infecciosas, causas externas e outras doenças.

Para avaliar a ingestão da dieta hospitalar durante a internação, foi aplicado pelo pesquisador um formulário adaptado de Proença et al., ${ }^{13}$ contendo questões pré-codificadas para investigar o grau de aceitação do paciente por tipo de refeição (pequenas: desjejum, colação, lanche e ceia; e grandes: almoço e jantar) durante o tempo de internação. Os indicadores utilizados para avaliar a influência na aceitação da dieta segundo esse instrumento foram: apresentação, sabor, temperatura e quantidade de refeição servida. Por conveniência do pesquisador e por apresentar maior percentual de distribuição no HUPE, o almoço foi a refeição escolhida para mensurar a quantidade de alimento ingerida pelo paciente, utilizando a técnica da observação direta. A estimativa visual do que restou na embalagem de alumínio individual foi registrada da seguinte forma: nada (0), menos da metade $(<1 / 2)$, igual ou mais da metade $(\geq 1 / 2)$ ou tudo $(1) .{ }^{6}$ A avaliação da dieta foi considerada favorável quando a opinião da refeição pelo paciente foi de "boa" a "ótima" e a ingestão da dieta servida foi acima da metade da quantidade servida.

A classe socioeconômica dos participantes foi avaliada segundo o Critério de Classificação Econômica Brasil. Para fins da análise estatística, a renda familiar foi reagrupada em classes: muito baixa (D e E), baixa (C1 e C2), média (B1 e B2) e alta (Ale A2).

Dados referentes ao grau de aceitação em relação à dieta foram tabulados no programa estatístico Epinfo 6.04 e analisados pelo software SPSS 16.0 (Statistical Package for the Social Sciences, Chicago). Foi considerado risco a (alfa) menor ou igual a 5\%. O estudo foi aprovado pelo Comitê de Ética em Pesquisa do Hospital Universitário Pedro Ernesto (protocolo n 2.712, aprovado em 5 de agosto de 2010). Cada entrevistado foi informado sobre a necessidade da assinatura de um Termo de Consentimento Livre e Esclarecido e da garantia de sigilo sobre as informações obtidas.

\section{Resultados}

Foram selecionados 145 pacientes para o estudo, sendo três excluídos (por desistência) e dez perdidos (ausência de resposta ou inconsistência de informações), totalizando 132 pacientes efetivamente estudados. De forma geral, a dieta hospitalar foi avaliada como de boa a ótima em $59,8 \%$ dos casos. Ao se analisar por tipo de refeição, verificou-se que as pequenas refeições foram mais bem aceitas que o almoço e o jantar $(\mathrm{p}<0,001)$, segundo a tabela 1 . 
Quanto à população investigada, verificou-se que mais da metade dos pacientes eram adultos acima dos 45 anos $(63,7 \%)$, do sexo masculino $(56,1 \%)$, portadores de doenças crônicas $(65,9 \%)$ e pertenciam à classe econômica baixa e muito baixa (78,9\%). Inicialmente, foi verificado que as variáveis sociodemográficas não influenciaram na aceitação da dieta (tabela 1). Porém, ao analisar por tipo de refeição, verificou-se que no almoço os pacientes da classe baixa relataram melhor aceitação da dieta, em comparação aos pacientes da classe média $(\mathrm{p}=0,02)$.

Tabela 1. Avaliação da aceitabilidade da dieta segundo características sociodemográficas e tipo de refeição. Rio de Janeiro-RJ, 2010.

\begin{tabular}{|c|c|c|c|}
\hline & \multicolumn{2}{|c|}{ Número (\%) de sujeitos: } & \multirow{2}{*}{$p^{1}$} \\
\hline & Regular a Ruim & Boa a Ótima & \\
\hline \multicolumn{4}{|l|}{ Sexo } \\
\hline Masculino & $32(43,2)$ & $21(36,2)$ & \multirow[t]{2}{*}{0,41} \\
\hline Feminino & $42(56,8)$ & $37(63,8)$ & \\
\hline \multicolumn{4}{|l|}{ Faixa etária } \\
\hline $18-29$ & $5(62,5)$ & $3(37,5)$ & \multirow{4}{*}{0,25} \\
\hline $30-45$ & $19(47,5)$ & $21(52,5)$ & \\
\hline $46-60$ & $17(37,8)$ & $28(62,2)$ & \\
\hline+60 & $12(30,8)$ & $27(69,2)$ & \\
\hline \multicolumn{4}{|l|}{ Tipo de patologia } \\
\hline Doenças infecciosas & $5(33,3)$ & $10(66,7$ & \multirow{5}{*}{0,46} \\
\hline Doenças crônicas & $37(42,5)$ & $50(57,5)$ & \\
\hline Outras causas & $7(50,0)$ & $7(50,0)$ & \\
\hline Causas externas & $4(25,0)$ & $12(75,0)$ & \\
\hline Média & $14(50,0)$ & $14(50,0)$ & \\
\hline \multicolumn{3}{|l|}{ Classe econômica ${ }^{-}$} & \multirow{3}{*}{0,21} \\
\hline Baixa & $24(33,3)$ & $48(66,7)$ & \\
\hline Muito baixa & $15(46,9)$ & $17(53,1)$ & \\
\hline \multicolumn{4}{|l|}{ Tipo de refeições ${ }^{-}$} \\
\hline Pequenas & $87(61,9)$ & $45(34,1)$ & \multirow[t]{2}{*}{$<0,001$} \\
\hline Grandes & $71(53,8)$ & $61(46,2)$ & \\
\hline
\end{tabular}

${ }^{1}$ Teste Qui-quadrado 
Quanto ao tipo de dieta, a maioria dos pacientes (66,7\%) recebeu dieta com algum tipo de restrição de nutriente (modificada) e, dentre essas, 61 (68,9\%) foram representados pela dieta hipossódica. Constatou-se que embora houvesse tendência a maior aceitação da dieta modificada do que a dieta básica pelos pacientes, essa diferença só foi estatisticamente significativa nas pequenas refeições (tabela 2).

Tabela 2. Avaliação das refeições segundo as características qualitativas da dieta. Rio de Janeiro-RJ, 2010.

\begin{tabular}{|c|c|c|c|c|}
\hline \multicolumn{2}{|c|}{ Refeição } & \multicolumn{2}{|c|}{$\mathrm{n}(\%)$} & \\
\hline \multicolumn{2}{|c|}{ Tipo de dieta } & Regular a Ruim & Boa a Ótima & $p^{1}$ \\
\hline \multirow[t]{2}{*}{ Desjejum } & Básica & $14(31,8)$ & $30(68,2)$ & \multirow{2}{*}{0,33} \\
\hline & Modificada & $21(23,9)$ & $67(76,1)$ & \\
\hline \multirow[t]{2}{*}{ Colação } & Básica & $21(47,7)$ & $23(52,3)$ & \multirow{2}{*}{0,00} \\
\hline & Modificada & $19(21,6)$ & $69(78,4)$ & \\
\hline \multirow[t]{2}{*}{ Almoço } & Básica & $19(43,2)$ & $25(56,8)$ & \multirow{2}{*}{0,61} \\
\hline & Modificada & $34(33,6)$ & $54(65,4)$ & \\
\hline \multirow[t]{2}{*}{ Lanche } & Básica & $18(40,9)$ & $26(59,1)$ & \multirow{2}{*}{0,00} \\
\hline & Modificada & $14(15,9)$ & $74(84,1)$ & \\
\hline \multirow[t]{2}{*}{ Jantar } & Básica & $17(38,6)$ & $27(61,4)$ & \multirow{2}{*}{1,00} \\
\hline & Modificada & $34(38,6)$ & $54(61,4)$ & \\
\hline \multirow[t]{2}{*}{ Ceia } & Básica & $27(61,4)$ & $17(38,6)$ & \multirow{2}{*}{0,01} \\
\hline & Modificada & $32(36,4)$ & $56(63,4)$ & \\
\hline
\end{tabular}

${ }^{1}$ Teste Qui-quadrado

Quanto ao teor de sal na dieta, não houve diferença estatística entre a aceitação da dieta normossódica e a hipossódica nas grandes refeições, diferentemente do observado em relação às pequenas refeições (colação: $p=0,02$; lanche: $p=001$; e ceia: $p=0,03$ ). 
Por meio da técnica de observação direta, a aceitação do almoço foi avaliada e confrontada com as respostas dos questionários. Os resultados revelaram que 88,7\% dos pacientes apresentaram ingestão igual ou de mais da metade do total da refeição (tabela 3). Houve correlação entre os dois métodos utilizados, pois aqueles que avaliaram o almoço como de bom a ótimo foram os que consumiram mais da metade da refeição $(r=0,38 ; \mathrm{p}<0,001)$.

Tabela 3. Percentual do grau de aceitação do almoço segundo a pesquisa de satisfação dos pacientes. Rio de Janeiro-RJ, 2010.

\begin{tabular}{|c|c|c|c|c|}
\hline \multirow{2}{*}{$\begin{array}{l}\text { Consumo de } \\
\text { dieta }\end{array}$} & \multirow{2}{*}{$\begin{array}{l}\text { Total consumo } \\
\qquad \mathrm{n}(\%)\end{array}$} & \multicolumn{2}{|c|}{ Aceitação n(\%) } & \multirow{2}{*}{$p^{1}$} \\
\hline & & Regular a Ruim & Boa a Ótima & \\
\hline$<1 / 2$ & $15(11,4)$ & $13(86,7)$ & $2(13,3)$ & \\
\hline$>1 / 2$ & $36(27,3)$ & $16(44,4)$ & $20(56,6)$ & $<0,001$ \\
\hline Tudo & $81(61,4)$ & $24(29,6)$ & $57(70,4)$ & \\
\hline
\end{tabular}

Teste Qui-quadrado

Entre os indicadores predeterminados que poderiam influenciar na aceitação da dieta hospitalar, a temperatura inadequada percebida foi o que mais contribuiu para a baixa ingestão das refeições servidas. Em menor proporção, a falta de tempero (sabor) das preparações dietéticas também foi considerada como fator responsável para a baixa aceitação nas grandes refeições, enquanto o horário de distribuição foi considerado para a ceia. Nessa instituição, a distribuição da ceia se faz juntamente com o jantar, devido ao número insuficiente de funcionários para cobrir o turno da noite. No desjejum, 22 pacientes (16,7\%); 33 (25\%) na colação; 13 (9,8\%) no almoço; cinco $(3,8 \%)$ no lanche; 29 (22\%) no jantar e $21(15,9 \%)$ na ceia relataram que nenhum dos indicadores supracitados interferiu na aceitação da refeição (tabela 4). 
Tabela 4. Percentual de fatores ambientais que afetaram de forma negativa ${ }^{1}$ a aceitação da dieta por tipo de refeição. Rio de Janeiro-RJ, 2010.

\begin{tabular}{ccccccc} 
& \multicolumn{3}{c}{ Pequenas Refeições } & \multicolumn{2}{c}{ Grandes Refeições } \\
\cline { 2 - 7 } & Desjejum & Colação & Lanche & Ceia & Almoço & Jantar \\
Apresentação & $3(2,3)$ & $2(1,5)$ & $3(2,3)$ & $0(0,0)$ & $4(3,0)$ & $1(0,8)$ \\
Horário & $1(0,8)$ & $5(3,8)$ & $19(14,4)$ & $64(48,5)$ & $6(4,5)$ & $49(37,1)$ \\
Sabor & $12(9,1)$ & $12(9,1)$ & $11(8,3)$ & $5(3,2)$ & $30(22,7)$ & $28(21,2)$ \\
Temperatura & $71(53,8)$ & $54(40,9)$ & $59(44,7)$ & $33(25,8)$ & $63(47,7)$ & $32(24,2)$ \\
Quantidade & $23(17,4)$ & $26(19,7)$ & $11(8,3)$ & $9(6,8)$ & $16(12,1)$ & $17(12,9)$ \\
\hline
\end{tabular}

${ }^{1} \mathrm{Na}$ tabela constam apenas os participantes que registraram algum fator ambiental que influenciasse de forma negativa a aceitação da dieta.

Do total dos entrevistados, $82,6 \%$ estavam internados há pelo menos duas semanas. O tempo de internação influenciou de forma negativa na aceitabilidade da dieta, visto que esta decresceu à medida que aumentou o tempo de internação, principalmente em relação ao almoço $(p<0,001)$ e ao jantar $(\mathrm{p}=0,02)$, como mostra a figura 1. Não houve diferença estatística em relação às pequenas refeições.

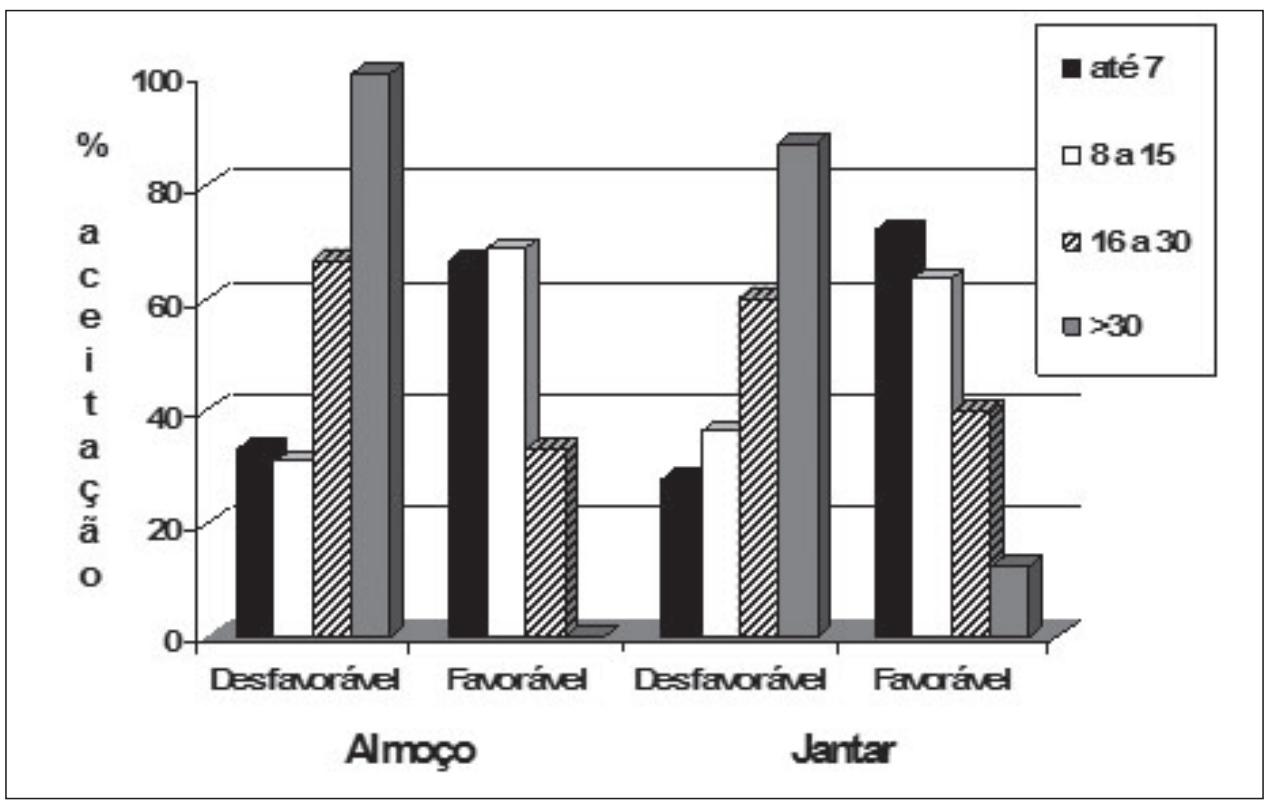

Figura 1. Relação entre aceitabilidade da dieta e dias de internação. 
Quanto às informações colhidas sobre o atendimento na enfermaria, 93\% dos entrevistados consideraram importante a apresentação pessoal dos copeiros e acrescentaram que os mesmos representam fonte de apoio, segurança e solidariedade no momento da alimentação.

Através da técnica de observação, o pesquisador pode perceber que a equipe de saúde colaborou para que os procedimentos médicos e de rotina não fossem realizados nos horários das refeições, embora algumas vezes fossem inevitáveis. Do total dos entrevistados, 98 (74,2\%) elogiaram a atenção, o carinho e todos os esclarecimentos prestados pela equipe de nutrição.

\section{Discussão}

Este trabalho se diferencia dos demais por avaliar a aceitação da dieta hospitalar não de forma isolada, mas estratificada por tipo de refeição (do desjejum à ceia). As autoras entendem que o alcance das necessidades nutricionais do paciente depende da aceitação de todas as refeições servidas e não somente das grandes refeições, como explorado em alguns trabalhos. ${ }^{14,15}$ Outro ponto importante foi investigar a relação do perfil dos usuários atendidos, no contexto em que os pacientes estão inseridos, com o processo de aceitação da dieta hospitalar.

Quanto ao grau de aceitação da dieta, observou-se que a avaliação foi de boa a ótima na maioria dos casos. Os resultados revelaram que a maioria dos pacientes apresentou ingestão de mais da metade do total da refeição e que houve correlação entre os dois métodos utilizados. Parte do sucesso se deve à integração da equipe de enfermagem, seja pelo respeito na realização de procedimentos fora dos horários das refeições, seja pelo atendimento nutricional prestado pelo serviço de nutrição clínica e dietética, conforme observado durante a distribuição.

Neste estudo, também se observou que as pequenas refeições tiveram melhor aceitação em relação às grandes refeições, exceto a ceia. Este fato é considerado importante para a equipe de nutrição, visto que nos lanches normalmente são realizadas as suplementações proteico-calóricas para recuperação nutricional dos pacientes criticamente enfermos. Ademais, observa-se que os lanches das dietas modificadas apresentam menor restrição do sabor, em comparação às grandes refeições, já que contam com a variedade de alimentos dietéticos existentes no mercado. No HUPE, no cardápio das pequenas refeições são permitidas opções de líquidos (mingau, leite, vitamina e mate) e os recheios são ofertados em porções individuais (geleia, margarina, queijo fundido), que favorecem a apresentação e a aceitação da refeição hospitalar.

Hiesmayr et al. ${ }^{16}$ comprovaram em seus resultados que a baixa ingestão alimentar é o maior fator de risco independente para a mortalidade hospitalar e que os lanches servidos e consumidos seriam fatores protetores para a mesma (razão de risco $=0,81$ para cada lanche). No estudo de Coloço et al. ${ }^{17}$ não foi encontrada diferença significativa entre a aceitação das pequenas e grandes refeições. 
No presente trabalho, as dietas com restrição de nutrientes (modificadas), incluindo a hipossódica, fornecidas durante a colação, lanche e ceia, apresentaram maior grau de aceitação entre seus usuários do que as dietas básicas e com sal. Sugere-se que os pacientes que recebem a dieta modificada são mais conscientes e interessados em seguir a alimentação preconizada para seu restabelecimento e, por isso, são menos críticos quanto a sua avaliação. A adição de ervas às preparações produzidas nesse hospital e a entrega de um saché de $1 \mathrm{~g}$ de sal aos pacientes em dieta hipossódica também foram consideradas possíveis fatores contribuintes para tais resultados. É importante acrescentar que embora as dietas modificadas tivessem sido mais bem avaliadas pelos pacientes, a literatura aponta que pacientes que recebem dietas modificadas normalmente alcançam as recomendações nutricionais, por serem dietas mais restritivas e menos palatáveis. ${ }^{6}$

Ao analisar o perfil de nossa clientela, constatou-se que a classe econômica foi a única variável sociodemográfica que demonstrou interferir no grau de satisfação da dieta, mesmo assim somente nas grandes refeições, contrastando com outros estudos, que demonstraram que gênero, idade e patologia são outros fatores que também influenciam na aceitação da dieta. ${ }^{17,18}$ Embora não tenha sido observada diferença estatística, constatou-se uma tendência dos pacientes mais jovens a rejeitarem a alimentação em relação aos mais velhos.

Em relação ao poder aquisitivo, observou-se que os pacientes de classes sociais mais baixas (D e E) tiveram maior aceitabilidade em comparação com os de classes mais favorecidas. Estudos indicam que os segmentos de renda mais alta tendem a estabelecer uma relação diferenciada com a alimentação, no sentido de valorizar a escolha de certos alimentos em detrimento de outros (90,91\%), seja pelo nível educacional mais elevado ou maior preocupação com a forma de apresentação. ${ }^{19}$

Quanto ao tempo de internação, Stanga et al. ${ }^{20}$ verificaram que pacientes com maiores períodos de internação apresentavam menor apetite, e consequentemente, menor aceitabilidade, o que foi comprovado neste estudo, sobretudo em relação às grandes refeições. Fato relevante, visto que o tempo de internação é diretamente proporcional ao gasto com a saúde e o grau de morbidade. ${ }^{3}$ Por isso, uma das metas do Sistema Único de Saúde (SUS) e desse hospital é diminuir o tempo médio de internação hospitalar e o sofrimento humano.

Entre os aspectos sensoriais e ambientais, constatou-se que a temperatura foi o que mais influenciou negativamente na aceitação da dieta. Uma razoável explicação se refere ao fato de que os carros de distribuição não são térmicos, assim como os utensílios que acondicionam as refeições. A longa distância e o grande número de leitos por copeira são outros fatores que podem ter contribuído para que a refeição servida à maior parte dos pacientes estivesse em temperatura percebida como inadequada.

Yabuta et al. ${ }^{14}$ mostraram que mais de $70 \%$ dos pacientes avaliados em seu estudo consideravam a temperatura da refeição o aspecto mais relevante no serviço. A temperatura (41,5\%) foi também 
o quesito mais influente na aceitação das refeições em estudos similares. ${ }^{15,20}$ Embora o horário de distribuição das refeições fosse outro fator ambiental de relevância que afetou a aceitação do jantar e da ceia, acredita-se que isso só foi mencionado devido ao problema de falta de funcionários do hospital, que faz com que a ceia seja servida junto com o jantar.

Diante do exposto, medidas como adequar o fracionamento, alterando o horário de distribuição da ceia, e o controle da temperatura na distribuição dos alimentos tornam-se essenciais para melhorar a aceitação alimentar, suprindo melhor as necessidades dos pacientes, o que poderá resultar no aumento da satisfação dos mesmos e na redução do tempo de hospitalização.

A reforma e a modernização da infraestrutura do serviço de nutrição e dietética, como a terceirização total dos serviços de alimentação, são algumas das estratégias que o serviço de nutrição e dietética desse hospital traçou para resolver os problemas com a temperatura e o horário da distribuição das refeições, que também se tornaram mais aparentes após a aplicação da pesquisa de aceitação nos pacientes internados no HUPE.

Conclui-se que a avaliação da dieta hospitalar deve ser realizada de forma contínua e abrangente, para que os fatores que influenciam na satisfação do usuário possam ser detectados e modificados, antes que comprometam seu estado nutricional e fisiopatológico.

\section{Referências}

1. Vanis N, Mesihovi R. Application of nutritional screening tests for determining prevalence of hospital malnutrition. Med Arch. 2008;62(4):211-4.

2. Venzin RM et al. How important is malnutrition? A prospective study in internal medicine. Eur J Clin Nutr. 2009;63(3):430-6.

3. Waitzberg DL, Caiaffa WT, Correia MI. Hospital malnutrition: the Brazilian national survey (IBRANUTRI): A study of 4000 patients. Nutrition 2001;17(7-8):573-80.

4. Elia, M. Nutrition, hospital food and in-hospital mortality. Clin Nutr. 2009;28(482):481-3.

5. Souza MD, Nakasato MA. Gastronomia hospitalar auxiliando na redução dos índices de desnutrição entre pacientes hospitalizados. Mundo saúde. 2011;35(2):208-14.

6. Dupertuis YM et al. Food intake in 1707 hospitalized patients: a prospective comprehensive hospital survey. Clin Nutr. 2003;22 (2):115-23.

7. Wright ORL, Conelly LB, Capra S. Consumer evaluation of hospital food service quality: An empirical investigation. Int J Health Care Qual Assur. 2006;19(2):181-94.

8. Stratton RJ, Elia M. A review of reviews: a new look at the evidence for oral nutrition supplements in clinical practice. Clin Nutr. 2007;(supl.2):5-23. 
9. Demário RL, Sousa AA, Salles RK. Comida de hospital: percepções de pacientes em um hospital público com proposta de atendimento humanizado. Ciênc saúde coletiva. 2010;15 (supl.1):1275-82.

10. Garcia RWD. A dieta hospitalar na perspectiva dos sujeitos envolvidos em sua produção e em seu planejamento. Rev Nutr. 2006;19(2):129-44.

11. Poulain JP. Sociologias da alimentação: os comedores e o espaço social alimentar. Florianópolis : Editora da UFSC; 2004.

12. Sousa AA, Proença RPC. La gestion des soins nutritionnels dans le secteur hospitalier: une etude comparative Bresil-France. Rech Soins Infirm. 2005;(83):28-33.

13. Proença RPC et al. Qualidade nutricional e sensorial na produção de refeições. Florianópolis: UFSC; 2005.

14. Yabuta CY, Cardoso E, Isosaki M. Dieta hipossódica: aceitação por pacientes internados em hospital especializado em cardiologia. Rev Bras Nutr Clin. 2006; 21(1): 33-7.

15. Sousa AA, Gloria MS, Cardoso TS. Aceitação de dietas em ambiente hospitalar. Rev Nutr. 2011; 24(2):287-94.

16. Hiesmayr M et al. The Nutrition Day Audit Team. Decreased food intake is a risk factor for mortality in hospitalised patients: the Nutrition Day survey 2006. Clin Nutr. 2009; 28:484-91.

17. Coloco RB, Holanda LB, Portero-Mcleilan KC. Determinantes do grau de satisfação de pacientes internados referente a refeições oferecidas em um hospital universitário. Rev. Ciênc. Med. 2009;18(3):121-30.

18. Zazzo JF, Puissant MC, Aubert P. Nutritional status and food intake in adult, pediatric and elderly hospitals: a 4-days survey in Assistance Publique-Hôpitaux de Paris. Nutr Clin Metabol. 2003; 17(4):213-17.

19. Lifchitz J. Alimentação e cultura: em torno ao natural. Physis. 1997;7:69-83.

20. Stanga Z et al. Hospital food: a survey of patients perceptions. Clin Nutr. 2003;22(3):241-6.

Recebido: 14/9/2013

Revisado: 09/3/2013

Aprovado: 23/5/2013 